\title{
Intermittent fasting modulation of the diabetic syndrome in sand rats. III. Post-mortem investigations
}

\author{
LOUIZA BELKACEMI ${ }^{1}$, GHALEM SELSELET-ATTOU ${ }^{1}$, NURDAN BULUR $^{2}$, \\ KARIM LOUCHAMI $^{2}$, ABDULLAH SENER $^{2}$ and WILLY J. MALAISSE ${ }^{2}$ \\ ${ }^{1}$ Laboratoire de Technologie Alimentaire et Nutrition, Université de Mostaganem, Mostaganem, Algeria; \\ ${ }^{2}$ Laboratory of Experimental Hormonology, Université Libre de Bruxelles, Brussels, Belgium
}

Received September 24, 2010; Accepted October 25, 2010

DOI: $10.3892 / \mathrm{ijmm} .2010 .556$

\begin{abstract}
The present report concerns several post-mortem variables examined in sand rats that were either maintained on a vegetal diet (control animals) or exposed first during a 20-day transition period to a mixed diet consisting of a fixed amount of a hypercaloric food and decreasing amounts of the vegetal food and then to a 30-day experimental period of exposure to the hypercaloric food. During the latter period, all animals were either given free access to food or fasting daily for 15 h, i.e. from 5.00 p.m. to 8.00 a.m. The body weight, liver wet weight, pancreas wet weight, plasma glucose and haemoglobin A1c concentration, plasma insulin concentration, insulinogenic index, insulin resistance HOMA, plasma cholesterol and triglyceride concentration, liver triglyceride and phospholipid content were all measured. Pancreatic islet (insulin, GLUT2) and liver (lipid droplets) histology were also examined. The main findings consisted in a lower body weight of fasting than non-fasting animals, a higher liver weight in non-diabetic and diabetic rats than in control non-fasting (but not so in fasting) animals, a decrease of pancreas weight in non-diabetic and diabetic as distinct from control animals, a fasting-induced decrease in plasma glucose, plasma insulin and insulin resistance HOMA, plasma cholesterol and triglyceride concentration and triglyceride liver content.
\end{abstract}

\section{Introduction}

Food excess and sedentary behaviour, characterizing modern society, cause many metabolic disturbances, especially in glucose and lipid homeostasis. These metabolic disorders

Correspondence to: Professor W.J. Malaisse, Laboratory of Experimental Hormonology, Université Libre de Bruxelles, 808 Route de Lennik, B-1070 Brussels, Belgium

E-mail: malaisse@ulb.ac.be

Key words: sand rats, intermittent fasting, body, liver and pancreas weight, plasma glucose, haemoglobin A1c, insulin, cholesterol and triglyceride, liver triglyceride and phospholipid, pancreatic insulin and GLUT2 participate to the development of insulin resistance in target organs. For instance, the liver accumulation of triglycerides resulting from high energy food intake is known to be associated with hepatic insulin resistance (1). The development of insulin resistance may also eventually lead to deterioration of insulin secretion, for instance as a result of pancreas exhaustion. The combination of these two disturbances results in type 2 diabetes or the metabolic syndrome, i.e. major metabolic diseases besides obesity.

Correction of feeding behaviour by decreasing food intake could have an opposite effect and could improve insulin resistance and/or insulin secretion, and consequently delay the consequences of perturbations in these variables.

The major aim of the present series of reports is to explore the possible effects of a daily intermittent fasting for $15 \mathrm{~h}$, i.e. from 5 p.m. to 8 a.m., upon the development of the diabetic syndrome provoked, in sand rats, by the transition from a vegetal diet to a hypercaloric one. In the first report, attention was drawn to such items as the biotope and type of vegetal diet of the sand rats (Psammomys obesus) in their desertic habitat, with emphasis on the daily pattern of food intake by these animals in their natural environment (2). The second report concerned mainly the time-related changes in body weight, postprandial glycemia and glucose tolerance provoked in the sand rats by the transition from the purely vegetal diet to the hypercaloric diet and the comparison between non-fasting and intermittently fasting animals during a further 30-day period of exposure to the hypercaloric diet (3). The present report deals with several variables examined when the animals were sacrified at the end of the latter period.

\section{Materials and methods}

The design of the present experiments conducted in 52 sand rats, up to the time of sacrifice, was already described in detail in two prior reports (2,3). Briefly, after capture and after 15 days of acclimatization, during which the sand rats had only access to a vegetal diet, they were, during a subsequent 20-day transition period either maintained on the same vegetal diet (14 control animals) or given access to a mixed diet composed of standard laboratory chow (20 g per animal per day) and decreasing amounts of the vegetal diet (representing $75 \%$ during the first week, $50 \%$ during the second week and $25 \%$ during the third week of animal body weight). In the 
Table I. Body, liver and pancreas weight.

\begin{tabular}{|c|c|c|c|c|c|}
\hline \multirow[b]{2}{*}{ Rats } & \multirow{2}{*}{$\begin{array}{c}\text { Body wt. } \\
\text { (g) }\end{array}$} & \multicolumn{2}{|c|}{ Liver wt. } & \multicolumn{2}{|c|}{ Pancreas wt. } \\
\hline & & (g) & (\% of body wt.) & $(\mathrm{mg})$ & (\%o body wt.) \\
\hline Control non-fasting & $127.5 \pm 4.2(10)$ & $4.03 \pm 0.18(10)$ & $3.19 \pm 0.16(10)$ & $316 \pm 37(10)$ & $2.49 \pm 0.29(10)$ \\
\hline Control fasting & $111.3 \pm 14.3(4)$ & $5.03 \pm 0.17(4)$ & $4.83 \pm 0.82(4)$ & $271 \pm 47(4)$ & $2.74 \pm 0.86(4)$ \\
\hline Non-diabetic non-fasting & $126.7 \pm 10.5(9)$ & $5.03 \pm 0.25(9)$ & $4.15 \pm 0.32(9)$ & $260 \pm 50(9)$ & $2.02 \pm 0.28(9)$ \\
\hline Non-diabetic fasting & $93.0 \pm 4.8(10)$ & $4.65 \pm 0.28(10)$ & $5.07 \pm 0.30(10)$ & $172 \pm 23(10)$ & $1.74 \pm 0.28(10)$ \\
\hline Diabetic non-fasting & $112.7 \pm 6.8(13)$ & $4.70 \pm 0.25(13)$ & $4.22 \pm 0.17(13)$ & $206 \pm 20(13)$ & $1.80 \pm 0.12(13)$ \\
\hline Diabetic fasting & $113.3 \pm 11.5(6)$ & $4.77 \pm 0.51(6)$ & $4.22 \pm 0.18(6)$ & $207 \pm 21(6)$ & $1.85 \pm 0.12(6)$ \\
\hline
\end{tabular}

latter case, the 38 sand rats were then divided in two groups of 19 animals each referred to as either non-diabetic (glycemia $<7.0 \mathrm{mM}$ ) or diabetic (glycemia $>8.3 \mathrm{mM}$ ). During the last 30 days of the present experiments, the animals were given access to either the vegetal diet (control animals) or the hypercaloric standard laboratory chow (diabetic and nondiabetic animals), some of the sand rats in each group undergoing intermittent fasting from 5 p.m. to 8 a.m. the next day. One day after the end of the experimental period of intermittent fasting, whenever applied, all sand rats were sacrificed in the postprandial state under anesthesia with ketamine chlorhydrate injected intraperitoneally. Blood was collected from the jugular vein in either heparinized tubes or in the presence of EDTA for haemoglobin A1c measurement.

The analytical methods used for the measurement of plasma glucose (4), hemoglobin A1c (5), insulin (6) were previously described in the cited references. Plasma cholesterol and triglycerides were measured by an ELITech (France) kit. Liver triglycerides (CHOD-PAP; Roche Diagnostic GmbH, Mannheim, Germany) and phospholipids (PAP 150; Biomerieux, Lyon, France) were measured in hepatic extracts (7), using the cited enzymatic kits.

For immunodetection of insulin, pancreatic rehydrated paraffin sections were blocked $1 \mathrm{~h}$ at room temperature with 1:20 normal goat serum (Vector Laboratories, Belgium) in PBS for non-specific reactions. The slides were incubated with primary anti-insulin (12018, Sigma-Aldrich, Belgium) mouse monoclonal antibody overnight at $4^{\circ} \mathrm{C}$ at a concentration of $1 / 3000$ in normal goat serum (1/20 in PBS). The secondary antibody, Rhodamine Red X-conjugated goat anti-mouse IgG (H+L) (115-295-146, Jackson ImmunoResearch Laboratories, USA) was applied at a dilution of $1 / 100$ in PBS/normal goat serum for $30 \mathrm{~min}$ at room temperature. The slides were mounted and DNA was counterstained with DAPI (Prolong ${ }^{\circledR}$ Gold antifade, Molecular Probes). The staining patterns were observed with an Axioplan and recorded with an Axiocam (Carl Zeiss, Germany).

For GLUT2 staining, immunodetection followed the standard ABC-DAB technique (8) using the avidin-biotin kit (Vector Laboratories). The slides were incubated overnight at $4^{\circ} \mathrm{C}$ with the first antibody: anti-Glut2 (sc-9117, Santa Cruz Biotechnology, Inc., CA, USA), a rabbit polyclonal antibody raised against amino acids 32-98 of GLUT2 of human origin. The primary antibody was used at dilutions $1 / 100$ to $1 / 1000$ in PBS with appropriate blocking serum at a dilution of 1/20. Purified immunoglobulins ( $\mathrm{IgG}$ ) (Sigma-Aldrich) from nonimmunized rabbit were used as negative controls. The slides were further incubated with the secondary biotinylated antibody: goat anti-rabbit IgG (H+L) (BA-1000, Vector Laboratories) at a dilution of 1/300 in PBS for $30 \mathrm{~min}$, at room temperature. The staining pattern was ascertained with an Axioplan and recorded with an Axiocam (Carl Zeiss).

For the histological study of the liver, hepatic samples were fixed in Bouin solution and embedded in paraffin. Sections $(5 \mu \mathrm{m})$ were deparrafined and rehydrated for hematoxylin-eosin staining. The liver slides were examined under light microscopy.

All results are presented as mean values $( \pm$ SEM) together with the number of individual observations (n) or degree of freedom (df). The statistical significance of differences between mean values was assessed by use of Student's t-test.

\section{Results}

Body, liver and pancreas weight. Whether in the fasting or non-fasting animals, the final body weight (wt.) failed to differ significantly in the control, diabetic and non-diabetic rats, with an overall mean value of $121.2 \pm 4.3 \mathrm{~g}(\mathrm{n}=32)$ in non-fasting animals, as distinct $(\mathrm{p}<0.02)$ from $102.8 \pm 5.2 \mathrm{~g}$ $(n=20)$ in fasting animals (Table I).

In absolute terms, the liver wet weight was higher in nonfasting diabetic rats $(4.70 \pm 0.25 \mathrm{~g} ; \mathrm{n}=13 ; \mathrm{p}<0.06)$ and nonfasting non-diabetic rats $(5.03 \pm 0.25 \mathrm{~g} ; \mathrm{n}=9 ; \mathrm{p}<0.005)$ than in non-fasting control animals $(4.03 \pm 0.18 \mathrm{~g} ; \mathrm{n}=10)$. Likewise, relative to body weight, the liver wet weight was significantly higher $(\mathrm{p}<0.02$ or less $)$ in non-fasting diabetic rats $(4.22 \pm 0.17 \% ; n=13)$ and non-diabetic rats $(4.15 \pm 0.32 \% ; n=9)$ than in non-fasting control rats $(3.19 \pm 0.16 \% ; n=10)$. In the fasting rats, the liver/body weight ratio averaged $121.4 \pm 7.2 \%$ $(n=20 ; p<0.004)$ of the mean corresponding values recorded in non-fasting animals of the same group (control, diabetic or non-diabetic), i.e. $100.0 \pm 3.0 \%(n=32)$. In absolute terms, however, the liver wet wt. failed to differ significantly $(\mathrm{p}>0.4)$ in fasting versus non-fasting animals, with respective overall mean values of $4.76 \pm 0.20 \mathrm{~g}(\mathrm{n}=20)$ and $4.59 \pm 0.15 \mathrm{~g}(\mathrm{n}=32)$.

In mirror image of the liver data, the wet weight of the pancreas was, in the non-fasting animals, lower $(\mathrm{p}<0.05)$ in the diabetic and non-diabetic rats $(227 \pm 24 \mathrm{mg} ; \mathrm{n}=22)$ than in 
Table II. Plasma glucose and insulin concentrations, insulinogenic index and insulin resistance HOMA at sacrifice.

\begin{tabular}{lcccc}
\hline Rats & $\begin{array}{c}\text { Plasma glucose } \\
(\mathrm{mM})\end{array}$ & $\begin{array}{c}\text { Plasma insulin } \\
(\mu \mathrm{g} / \mathrm{l})\end{array}$ & $\begin{array}{c}\text { Insulinogenic index } \\
(\mu \mathrm{g} / \mathrm{mol})\end{array}$ & $\begin{array}{c}\text { Insulin resistance HOMA } \\
(\mathrm{mM} . \mu \mathrm{g} / \mathrm{l})\end{array}$ \\
\hline Control non-fasting & $4.46 \pm 0.28(10)$ & $1.55 \pm 0.60(4)$ & $409 \pm 162(4)$ & $5.9 \pm 0.3(4)$ \\
Control fasting & $2.89 \pm 0.25(4)$ & $1.08 \pm 0.41(4)$ & $385 \pm 151(4)$ & $3.1 \pm 1.1(4)$ \\
Non-diabetic non-fasting & $4.61 \pm 0.45(9)$ & $2.95 \pm 0.37(4)$ & $653 \pm 110(4)$ & $13.4 \pm 1.1(4)$ \\
Non-diabetic fasting & $4.12 \pm 0.48(10)$ & $2.25 \pm 0.29(7)$ & $536 \pm 65(7)$ & $10.6 \pm 2.4(7)$ \\
Diabetic non-fasting & $16.56 \pm 1.52(13)$ & $9.35 \pm 2.34(8)$ & $599 \pm 135(8)$ & $156.0 \pm 45.6(8)$ \\
Diabetic fasting & $6.49 \pm 0.74(6)$ & $6.14 \pm 1.51(5)$ & $1,130 \pm 360(5)$ & $35.1 \pm 6.4(5)$ \\
\hline
\end{tabular}

the control rats $(316 \pm 37 \mathrm{mg} ; \mathrm{n}=10)$, such a difference being most marked $(\mathrm{p}<0.02)$ in the diabetic rats and failing to achieve statistical significance in the non-diabetic rats. The values recorded in the fasting rats averaged $80.5 \pm 6.7 \%$ $(n=20 ; p<0.08)$ of the corresponding mean values found in non-fasting rats of the same group (control, diabetic, nondiabetic), i.e. $100.0 \pm 7.3 \%(n=32)$. Likewise, when expressed relative to body weight, the pancreatic weight represented no more than $1.82 \pm 0.09$ and $1.88 \pm 0.19 \%$ ( $n=19$ in both cases) in diabetic and non-diabetic rats, as compared $(\mathrm{p}<0.04$ and $\mathrm{p}<0.06)$ to $2.56 \pm 0.30 \%$ o $(\mathrm{n}=14)$ in control animals.

Plasma glucose and HbAlc. At sacrifice, the plasma glucose concentration was comparable in non-fasting control animals $(4.46 \pm 0.28 \mathrm{mM} ; \mathrm{n}=10)$ and non-fasting non-diabetic rats $(4.61 \pm 0.45 \mathrm{mM} ; \mathrm{n}=9)$, and much higher $(\mathrm{p}<0.001)$ in nonfasting diabetic rats $(16.56 \pm 1.52 \mathrm{mM} ; \mathrm{n}=13)$. In the diabetic rats, it decreased $(\mathrm{p}<0.001)$ to $6.49 \pm 0.74 \mathrm{mM}(\mathrm{n}=6)$ in fasting animals. Likewise, in the control and non-diabetic rats, the mean value found in fasting animals $(3.77 \pm 0.38 \mathrm{mM} ; \mathrm{n}=14)$ represented no more than $82.4 \pm 8.1 \%(\mathrm{n}=14 ; \mathrm{p}<0.08)$ of the mean corresponding values $(100.0 \pm 5.6 \% ; n=19)$ recorded in non-fasting rats of the same group. As a matter of fact, such a decrease was highly significant $(\mathrm{p}<0.01)$ in the fasting control rats $(2.89 \pm 0.25 \mathrm{mM} ; \mathrm{n}=4)$, whilst failing to achieve statistical significance in the fasting non-diabetic rats $(4.12 \pm 0.48 \mathrm{mM}$; $\mathrm{n}=10$ ). The latter two mean values nevertheless failed to differ significantly from one another $(\mathrm{p}>0.1)$, and remained both lower ( $<<0.02$ or less) than that measured in the fasting diabetic rats.

In fair agreement with the plasma glucose data, the HbAlc measurements yielded much higher values $(\mathrm{p}<0.001)$ in non-fasting diabetic rats $(4.78 \pm 0.46 \% ; n=13)$ than in nonfasting control animals $(1.98 \pm 0.16 \% ; n=10)$. In the nonfasting non-diabetic rats, it averaged $2.73 \pm 0.33 \%(n=9)$ and, as such, was somewhat higher $(\mathrm{p}<0.05)$ than in non-fasting control animals but much lower $(\mathrm{p}<0.005)$ than in nonfasting diabetic rats. In the fasting diabetic rats the $\mathrm{HbA} 1 \mathrm{c}$ $(3.47 \pm 0.14 \% ; n=6)$ was somewhat lower $(\mathrm{p}<0.08)$ than in the non-fasting diabetic rats. However, such was not the case when comparing fasting to non-fasting animals in the other two groups of rats (control and non-diabetic). For instance, in the fasting non-diabetic rats, the HbA1c $(3.21 \pm 0.31 \%$; $=10)$ represented $117.4 \pm 11.4 \%$ of the corresponding mean value found in non-fasting non-diabetic rats, as distinct $(\mathrm{p}<0.01)$ from $72.5 \pm 2.9 \%(n=6)$ when comparing fasting diabetic rats to non-fasting diabetic rats.

Plasma insulin concentration, insulinogenic index and insulin resistance HOMA. Whether in intermittently fasting or nonfasting animals, the plasma insulin concentration was highest in the diabetic rats and lowest in the control animals (Table II). Thus, relative to the corresponding mean values found in diabetic rats $(100.0 \pm 17.4 \% ; n=13)$ examined under the same nutritional conditions (fasting or non-fasting), the measurements made in non-diabetic rats only represented $34.8 \pm 3.3 \%(\mathrm{n}=11 ; \mathrm{p}<0.005)$, an even lower value $(\mathrm{p}<0.005)$ being recorded in control animals $(17.1 \pm 3.7 \% ; n=8)$. The mean plasma insulin concentration was always lower in the intermittently fasting animals than in the non-fasting rats, the values recorded in the former animals representing $71.3 \pm 8.6 \%$ ( $n=16 ; \mathrm{p}<0.005$ versus unity) of the corresponding reference values found in the non-fasting rats $(100.0 \pm 15.1 \%$; $\mathrm{n}=16)$.

A different situation prevailed for the insulinogenic index, i.e. the ratio between plasma insulin and glucose concentrations (Table II). First, no significant difference was anymore observed between diabetic, non-diabetic and control rats. Indeed, relative to the corresponding mean values found in diabetic rats $(100.0 \pm 17.7 \% ; n=13)$, examined under the same nutritional conditions, the measurements made in nondiabetic and control rats averaged, respectively, $69.8 \pm 11.7 \%$ $(\mathrm{n}=11 ; \mathrm{p}>0.18$ versus diabetic rats) and $51.1 \pm 15.4 \%(\mathrm{n}=8$; $\mathrm{p}<0.08$ versus diabetic rats). The latter two percentages were both significantly higher than the corresponding values found for plasma insulin concentration in either the non-diabetic rats $(\mathrm{p}<0.01)$ or control animals $(\mathrm{p}<0.05)$. Second, no significant change in the insulinogenic index was anymore observed when comparing intermittently fasting to non-fasting rats. In the former rats, the insulinogenic index averaged (118.4 $\pm 23.2 \% ; n=16 ; p>0.4$ versus unity) of the corresponding reference values found in the non-fasting rats $(100.0 \pm 14.5 \%$; $\mathrm{n}=16$ ).

The HOMA for insulin resistance, taken as the product of plasma insulin concentration $(\mu \mathrm{g} / 1)$ times than glucose concentrations $(\mathrm{mM})$, only represented in the non-diabetic rats $21.4 \pm 5.2 \%(n=11 ; \mathrm{p}<0.001)$ of the mean corresponding values found in diabetic rats examined under the same nutritional conditions $(100.0 \pm 18.4 \% ; n=13)$. It was further decreased $(\mathrm{p}<0.03)$ to $6.6 \pm 1.7 \%(\mathrm{n}=8)$ of such mean values 

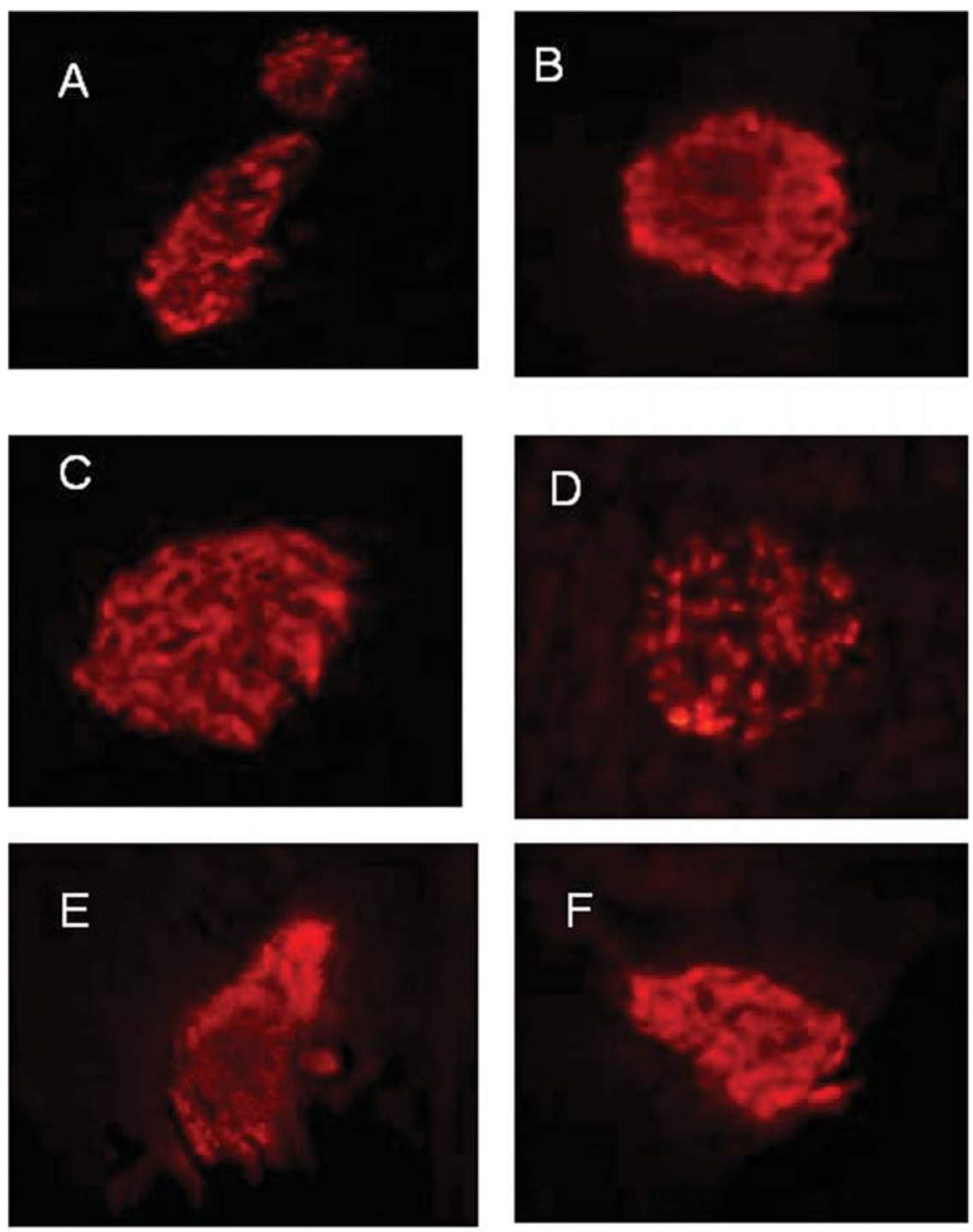

Figure 1. Immunodetection of insulin in the pancreas of fasting control (A), diabetic (C) and non-diabetic (E) and non-fasting control (B), diabetic (D) and non-diabetic $(\mathrm{F})$ sand rats. Immunohistochemical labelling was performed using fluorescein-labelled secondary antibody. Both manipulations were conducted at a 1:3000 dilution (magnification $\mathrm{x} 40$ ).

in the control animals. It averaged, in the intermittently fasting rats, $54.7 \pm 10.8 \%(\mathrm{n}=16 ; \mathrm{p}<0.01)$ of the mean corresponding values $(100.0 \pm 16.6 \% ; n=16)$ found in nonfasting rats in the same group of animals (control, non-diabetic or diabetic rats).

Plasma cholesterol and triglyceride concentration. Whether in the fasting or non-fasting rats, the plasma cholesterol concentration was significantly higher in the diabetic and non-diabetic rats than in the control ones. It failed to differ significantly in the diabetic and non-diabetic rats, whether in the fasting $(p>0.1)$ or non-fasting $(p<0.1)$ animals. It was significantly lower in the fasting than non-fasting rats in both the control animals $(\mathrm{p}<0.05)$ and diabetic rats $(\mathrm{p}<0.025)$, but not so in the non-diabetic rats. Pooling together all available data, it averaged in the fasting rats $82.6 \pm 5.2 \%(n=20 ; p<0.03)$ of the corresponding values found in the non-fasting animals of the same group (control, diabetic, non-diabetic), i.e. $100.0 \pm 5.1 \%(n=32)$.
The plasma triglyceride concentration was significantly higher $(\mathrm{p}<0.02$ or less $)$ in the non-fasting diabetic rats $(1,815 \pm 307 \mathrm{mg} / \mathrm{l} ; \mathrm{n}=13)$ and non-fasting non-diabetic rats $(2,041 \pm 397 \mathrm{mg} / \mathrm{l} ; \mathrm{n}=9)$ than in non-fasting control animals $(893 \pm 99 \mathrm{mg} / \mathrm{l} ; \mathrm{n}=10)$. It failed to differ significantly in the non-diabetic and diabetic rats, whether in the non-fasting ( $>0.6)$ or fasting ( $>>0.7)$ animals. It averaged 1,362 $\pm 254 \mathrm{mg} / \mathrm{l}$ $(\mathrm{n}=6)$ and $1,478 \pm 193 \mathrm{mg} / \mathrm{l}(\mathrm{n}=10)$ in the fasting diabetic and non-diabetic rats, respectively, representing 73.4 \pm 7.6\% ( $\mathrm{n}=16$; $\mathrm{p}<0.07$ ) of the mean corresponding values found in non-fasting rats of the same status (diabetic or non-diabetic animals), i.e. $100.0 \pm 10.2 \%(n=22)$. Such a decrease in the fasting versus non-fasting rats was not observed, however, in the control rats.

Liver triglyceride and phospholipid content. Whether in nonfasting or intermittently fasting rats, the liver triglyceride content was higher in non-diabetic and diabetic animals than in the control ones (Table III). It averaged in the former 
Table III. Lipid data.

\begin{tabular}{lccrl}
\hline Rats & $\begin{array}{c}\text { Plasma cholesterol } \\
(\mathrm{mg} / \mathrm{l})\end{array}$ & $\begin{array}{c}\text { Plasma triglycerides } \\
(\mathrm{mg} / \mathrm{l})\end{array}$ & $\begin{array}{c}\text { Liver TG }^{\mathrm{a}}(\mu \mathrm{mol} / \mathrm{g}) \\
\text { (1) }\end{array}$ & $\begin{array}{c}\text { Liver PL }^{\mathrm{b}} \\
(\mu \mathrm{mol} / \mathrm{g})\end{array}$ \\
\hline Control non-fasting & $607 \pm 57(10)$ & $893 \pm 99(10)$ & $3.52 \pm 0.56(4)$ & $20.29 \pm 0.94(4)$ \\
Control fasting & $398 \pm 28(4)$ & $1,185 \pm 575(2)$ & $2.24 \pm 0.24(4)$ & $13.15 \pm 2.70(4)$ \\
Non-diabetic non-fasting & $1,217 \pm 111(9)$ & $2,041 \pm 397(9)$ & $12.41 \pm 2.86(4)$ & $21.67 \pm 1.63(4)$ \\
Non-diabetic fasting & $1,211 \pm 78(10)$ & $1,478 \pm 193(10)$ & $5.16 \pm 0.74(4)$ & $19.07 \pm 0.94(4)$ \\
Diabetic non-fasting & $1,558 \pm 138(13)$ & $1,815 \pm 307(13)$ & $14.85 \pm 8.92(4)$ & $20.90 \pm 1.61(4)$ \\
Diabetic fasting & $1,025 \pm 72(6)$ & $1,362 \pm 254(6)$ & $6.29 \pm 1.39(4)$ & $20.42 \pm 0.47(4)$ \\
\hline
\end{tabular}

${ }^{a}$ The liver triglyceride content is expressed as $\mu \mathrm{mol}$ glycerol per $\mathrm{g}$ liver wet wt. ${ }^{\mathrm{b}}$ The liver phospholipid content is expressed as $\mu$ mol choline per g liver wet wt.

animals $321.6 \pm 92.2 \%(n=16)$ of the mean corresponding values found under the same experimental conditions (nonfasting or fasting) in the latter animals $(100.0 \pm 8.9 \% ; n=8)$. It averaged in the intermittently fasting rats $49.3 \pm 5.0 \% \quad(n=12$; $\mathrm{p}<0.02$ ) of the mean corresponding values found in non-fasting rats $(100.0 \pm 19.5 \% ; n=12)$. No significant difference was found between the non-diabetic and diabetic rats, whether in non-fasting rats or intermittently fasting animals.

As judged from the measurement of choline, the sole significant difference in the liver phospholipid content consisted in a lower value $(\mathrm{p}<0.05)$ found in the intermittently fasting as compared to non-fasting control rats (Table III).

Pancreatic islet histology. The staining for insulin was comparable in control, non-diabetic and diabetic sand rats (Fig. 1). It appeared somewhat less marked in fed than in fasting rats of the same group (control, diabetic, non-diabetic). There was no obvious difference for the GLUT2 staining in the different types of sand rats (Fig. 2).

Liver histology. No lipid droplet was observed in the liver of control sand rats, whether in non-fasting or fasting animals (Fig. 3). However, in both diabetic and non-diabetic sand rats, obvious accumulation of lipid droplets was observed this being more pronounced in non-fasting than in fasting animals (Fig. 3).

\section{Discussion}

The present study documents both the undesirable effects in sand rats or a hypercaloric diet, as distinct from a purely vegetal diet, upon selected metabolic and hormonal variables, and the beneficial effects of intermittent fasting upon the same variables in the animals exposed to the hypercaloric diet.

Glucose homeostasis. The present measurements of plasma glucose concentration extend our prior observations (3) on the improvement of glucose homeostasis in the diabetic sand rats undergoing intermittent fasting. These postprandial measurements are likely to reflect the long-term regulation of glycemia. There was indeed a close parallelism between the mean values for plasma glucose concentration and haemoglobin A1c. For instance, in the non-fasting or fasting diabetic and non-diabetic sand rats, there was a highly significant positive correlation $(\mathrm{r}=+0.6751$; $\mathrm{n}=38$; $\mathrm{p}<0.001)$ between these two variables. The results listed in Table II indicate that the fasting-induced decrease of glycemia could not be attributed to a higher rate of insulin secretion. On the contrary, the mean plasma insulin concentration was always lower in fasting sand rats than in non-fasting animals of the same group (control, non-diabetic, diabetic).

The improvement of glucose homeostasis caused by intermittent fasting appears, however, largely attributable to a decrease of insulin resistance, otherwise prevailing in the diabetic sand rats exposed to the hypercaloric diet. This proposal is supported by the tight correlation $(r=+0.7454$; $\mathrm{n}=32 ; \mathrm{p}<0.001$ ) found between the postprandial plasma glucose concentration and HOMA for insulin resistance (logarithmic values). It could obviously be objected that such a correlation is not meaningful since one of the two variables under consideration, i.e. the plasma glucose concentration, is taken into consideration to calculate the other variable, i.e. the HOMA for insulin resistance. Therefore, the HOMA for insulin resistance (logarithmic values) was compared to the haemoglobin A1c. In the 28 sand rats in which these two variables had been measured, a significant positive correlation $(\mathrm{r}=+0.4320 ; \mathrm{n}=28 ; \mathrm{p}<0.03)$ was again observed, thus reinforcing the view that insulin resistance was indeed a significant determinant of the perturbation of glucose homeostasis in the present experiments.

Body weight. The present study also documents that, in sand rats, intermittent fasting for $15 \mathrm{~h}$ over a period of 30 days eventually results in lower values for body weight, whether in control animals maintained on a vegetable diet or in rats exposed to a hypercaloric diet. In this respect, the present results closely resemble those collected in the same animals a week before sacrifice (3). For instance, in the animals exposed to the hypercaloric diet, the measurements of body weight averaged $110.0 \pm 4.0 \mathrm{~g}(\mathrm{n}=38)$ a week before sacrifice and $110.9 \pm 4.4 \mathrm{~g}(\mathrm{n}=38)$ at sacrifice, with a correlation coefficient between the two series of measurements amounting to +0.9094 $(\mathrm{n}=38 ; \mathrm{p}<0.001)$. It should be stressed, however, that no 

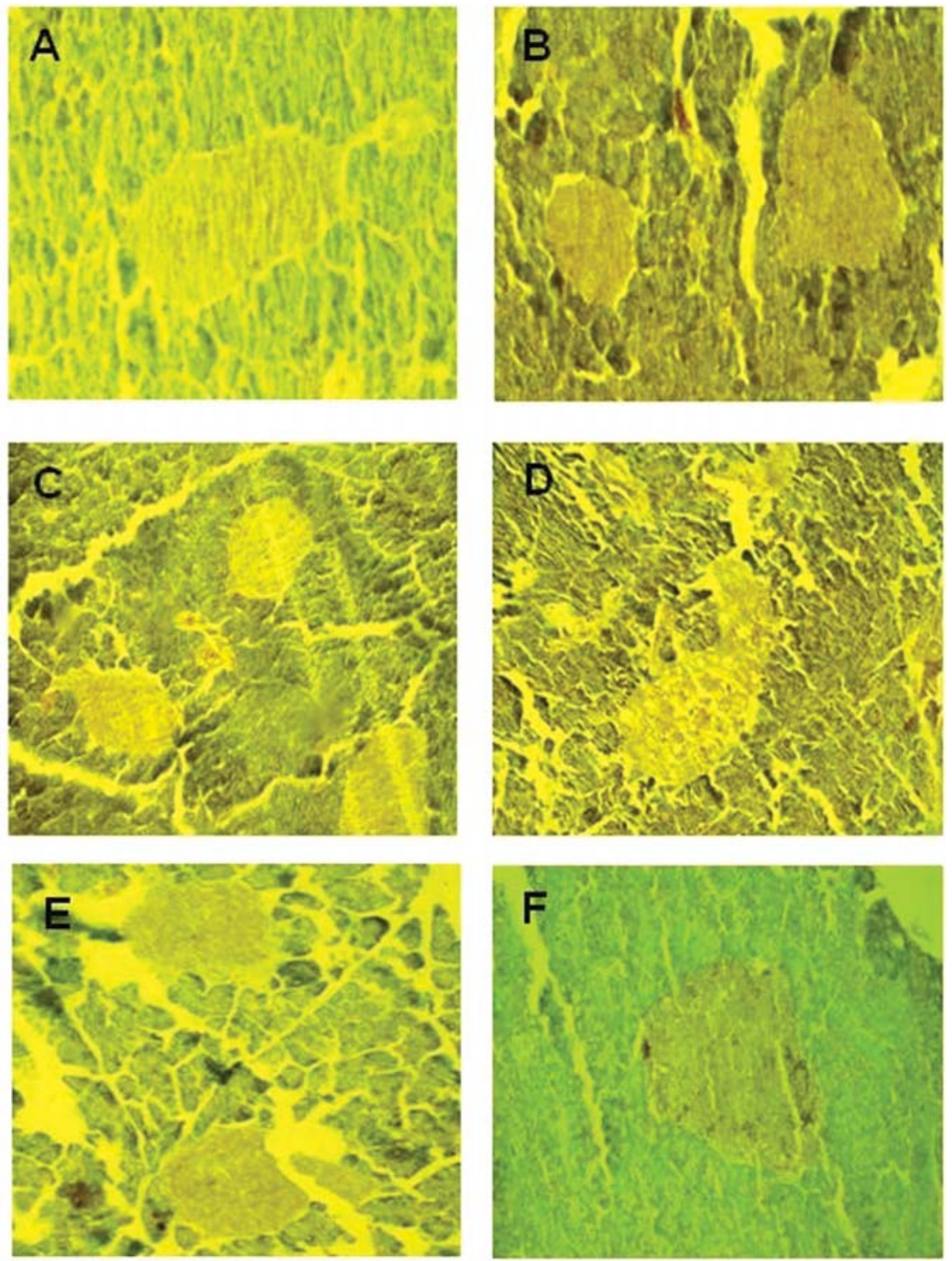

Figure 2. Immunodetection of GLUT2 by anti-GLUT2 (1/100) in the pancreas of fasting control (A), diabetic (C) and non-diabetic (E) and non-fasting control (B), diabetic (D) and non-diabetic (F) sand rats. Immunohistochemical labelling was performed using avidin-biotin-peroxidase complex with diaminobenzidine (magnification $\mathrm{x} 40$ ).

significant difference in body weight was found between non-diabetic or diabetic sand rats as compared to control animals, whether in the fasting or non-fasting groups $(\mathrm{p}>0.1$ or more).

Lipid variables. The exposure to the hypercaloric diet and intermittent fasting also affected lipid variables in both liver and plasma. Thus, despite virtually identical liver phospholipid content, the mean triacylglycerol hepatic content, expressed relative to liver wet weight, was much higher ( $<<0.03$, comparison of mean geometric values) in the nonfasting sand rats exposed to the hypercaloric diet $(10.19 \pm 2.82 \mu \mathrm{mol} / \mathrm{g} ; \mathrm{n}=8)$ than in the non-fasting control animals $(3.37 \pm 0.59 \mu \mathrm{mol} / \mathrm{g} ; \mathrm{n}=4)$. This situation prevailed despite the fact that both the absolute values for liver wet weight and the liver/body weight ratios were higher $(\mathrm{p}<0.01)$ in non-fasting rats exposed to the hypercaloric diet $(4.84 \pm 0.18 \mathrm{~g}$ and $4.19 \pm 0.16 \% ; \mathrm{n}=22)$ than in the non-fasting control animals $(4.03 \pm 0.18 \mathrm{~g}$ and $3.19 \pm 0.16 \% ; \mathrm{n}=10)$. The higher triglycerides accumulation in liver was also confirmed by histological examination of liver (Fig. 3). In fact, we observed a large number of lipid droplets in hepatic section which may represent the non-alcoholic steatohepatitis.

In fasting rats, the liver triglyceride content was also higher $(\mathrm{p}<0.001)$ in the diabetic and non-diabetic animals $(5.48 \pm 0.63 \mu \mathrm{mol} / \mathrm{g} ; \mathrm{n}=8)$ than in the control ones $(2.20 \pm 0.25 \mu \mathrm{mol} / \mathrm{g} ; \mathrm{n}=4)$. In all cases, the intermittent fasting decreased the liver triglyceride content, the values recorded in the fasting rats representing, on average, only half of those found in non-fasting animals of the same type (control, diabetic, non-diabetic). This coincided, especially in diabetic and non-diabetic sand rats, with a decreased number and size of lipid droplets in the liver of fasting animals as compared to non-fasting animals. Thus, intermittent fasting minimized the hepatic steatosis otherwise caused by high energy feeding.

These changes were, as a rule, paralleled by comparable changes in plasma triglyceride concentration. Thus, such a 

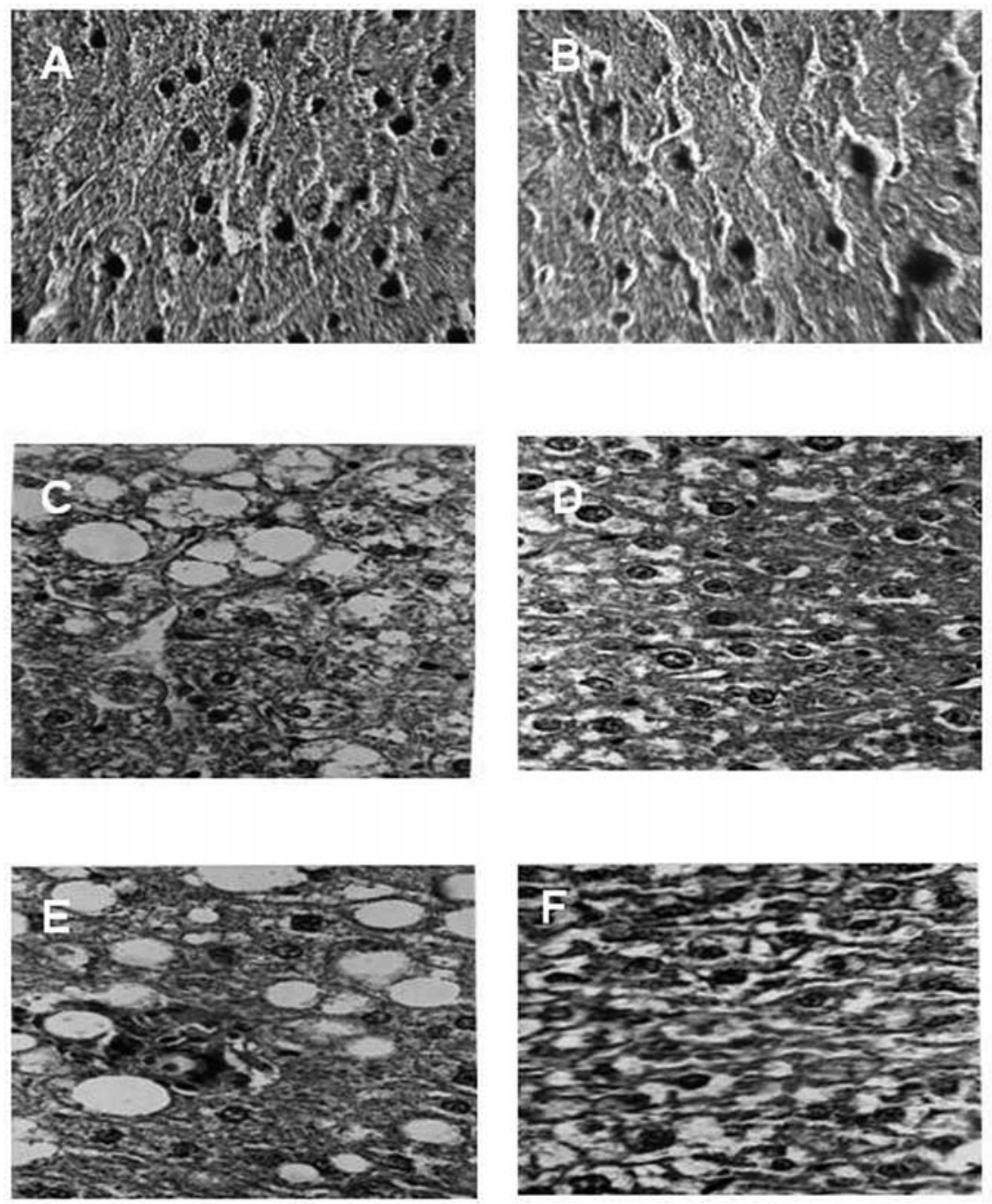

Figure 3. Hematoxylin-eosin stained liver histological slides obtained from non-fasting control (A), diabetic (C) and non-diabetic (E) sand rats, and fasting control (B), diabetic (D) and non-diabetic (F) animals (magnification x40).

concentration was higher in non-fasting diabetic and nondiabetic sand rats than in non-fasting control animals. In the former sand rats, it was decreased by intermittent fasting. Moreover, when considering all sand rats for which both the liver triglyceride content and plasma triglyceride concentration were available, a highly significant positive correlation $(r=+0.6536 ; n=22 ; \mathrm{p}<0.001)$ was found between these two variables.

Likewise, the plasma cholesterol concentration was higher in diabetic and non-diabetic sand rats than in control animals, whether in fasting or non-fasting animals. It was about $20 \%$ lower $(\mathrm{p}<0.03)$ in fasting sand rats than in nonfasting animals of the same type (control, diabetic, nondiabetic).

The accumulation of triglycerides in the liver is closely associated with hepatic insulin resistance (1), there being an inverse relationship between hepatic triglyceride content and hepatic insulin sensitivity (9). The decrease in hepatic triglyceride content in fasting sand rats could thus improve hepatic insulin sensitivity and, by doing so, contribute to the decrease in insulin resistance observed in this study in the same animals. Since hepatic insulin resistance coincides with a lesser sensitivity of the liver to the suppressive effects of insulin on hepatic glucose and VLDL production (10), the reduction of non-alcoholic steatohepatitis after the 30 days of intermittent fasting may also contribute to the concomitant improvement of hyperglycemia and hyperlipidemia. In turn, the improvement of glucose homeostasis may participate in a decrease of cholesterol synthesis. According to Scoppola et al (11), there is indeed a positive correlation between glycated haemoglobin concentration and the urinary excretion of mevalonate, taken as an indicator of cholesterol synthesis. The decrease in cholesterol synthesis may also involve the relationship between insulin sensitivity and cholesterol absorption, as proposed by Simonen et al (12).

Pancreatic islet functional and morphological status. In both non-fasting and fasting sand rats, the plasma insulin concentration was higher in non-diabetic animals than in control ones, and further increased in diabetic sand rats. It was always lower in fasting rats than in non-fasting animals. As already mentioned, this suggests that neither the deterioration of glucose homeostasis in sand rats exposed to the hypercaloric diet nor its improvement in intermittently fasting animals may be primarily attributable to changes in insulin output. 
The insulinogenic index, i.e. the ratio of plasma insulin/ glucose concentrations, was calculated to assess the secretory responsiveness of insulin-producing cells to the hexose. In the euglycemic control and non-diabetic sand rats, it only represented $61.9 \pm 9.3 \%(n=19)$ of the mean corresponding values $(100.0 \pm 17.7 \% ; n=13)$ found in diabetic rats examined in the same nutritional status (non-fasting or fasting animals). Such a difference $(p<0.05)$ is reminiscent of the increased insulin output from pieces of pancreatic tissue, when comparing sand rats exposed to a vegetable diet or remaining euglycemic when exposed to a mixed diet of vegetables and usual laboratory chow to moderately hyperglycaemic sand rats also exposed to the mixed diet (13). As a matter of fact, there was a close to perfect correlation $(r=+0.9841)$ between the mean insulinogenic index in the latter 3 groups of sand rats and the corresponding values found in the present study in control, non-diabetic and diabetic animals. The results collected in the latter diabetic animals represent a first indication that, under the present experimental conditions, the diabetic sand rats had not yet reached the later stage of impaired $\beta$-cell function documented in prior studies $(13,14)$.

It should be stressed that, in the fasting animals, the insulinogenic index was not significantly lower than in the non-fasting sand rats of the same group (control, non-diabetic and diabetic). As a matter of fact, the trend, if any, was, especially in the diabetic sand rats, towards an increase of the insulinogenic index. Thus, after logarithmic conversion of all available data, the values recorded in the fasting sand rats averaged $151.1 \pm 54.5 \%(\mathrm{df}=26)$ of the mean corresponding values recorded in non-fasting animals of the same group. This is an important finding. It documents that the intermittent fasting failed to affect adversely the insulin secretory responsiveness to glucose, unlike the severe reduction in such a responsiveness otherwise observed after starvation for one or more days (15).

The present histological documents also indicate that the ß-cells of non-fasting or fasting diabetic sand rats displayed no sizeable reduction of insulin content, at variance with the situation found in more severely diabetic sand rats (13). The immunodetection of GLUT2 also failed to reveal any major anomaly in the diabetic sand rats.

In conclusion, the present study documents that, in sand rats exposed to a hypercaloric diet, intermittent fasting improves glucose homeostasis, apparently mainly through a decrease in insulin resistance, decreases body weight, opposes the liver steatosis, hypertriglyceridemia and hypercholesterolemia otherwise found in sand rats exposed to the hypercaloric diet, and fails to alter both the insulin secretory responsiveness to glucose and insulin content of pancreatic islets. To the extent that these findings might be extrapolated to human subjects, with type II diabetes or prone to develop such a disease, the present intermittent fasting procedure, which is somehow reminiscent of the daily fasting period during the Ramadan, may well be recommended as a suitable approach to prevent, minimize or correct the metabolic and hormonal defects found in such subjects.

\section{Acknowledgments}

This study was supported in part by a grant from the Belgian Foundation for Scientific Medical Research (3.4520.07). We are grateful to A. Chwalik, P. D'Hont and A. Dufour for technical assistance and C. Demesmaeker for secretarial help.

\section{References}

1. Yki-Jarvinen $\mathrm{H}$ : Fat in the liver and insulin resistance. Ann Med 37: 347-356, 2005.

2. Belkacemi L, Selselet-Attou G, Sener A and Malaisse WJ: Intermittent fasting modulation of the diabetic syndrome in sand rats. I. Background information and experimental design. Metab Funct Res Diab 2: 5-8, 2009.

3. Belkacemi L, Selselet-Attou G, Louchami K, Sener A and Malaisse WJ: Intermittent fasting modulation of the diabetic syndrome in sand rats. II. In vivo investigations. Int J Mol Med 26: 759-765, 2010.

4. Bergmeyer HU and Berndt E: Glucose determination with glucose oxidase and peroxidase. In: Methods of Enzymatic Analysis. Bergmeyer HU (ed). Academic Press, New York, pp1205-1215, 1974.

5. Ersser RS, Barlow GB, Drew RG and Hjelm M: Packing materials suitable for rapid, analytical, low-pressure chromatography of hemoglobins on midget columns. Biomed Chromatogr 1: $183-188,1986$.

6. Leclercq-Meyer V, Marchand J, Woussen-Colle MC, Giroix M-H and Malaisse WJ: Multiple effects of leucine on glucagon, insulin and somatostatin secretion from the perfused rat pancreas. Endocrinology 116: 1168-1174, 1985.

7. Folch J, Lees M and Sloane-Stanley GH: A simple method for the isolation and purification of total lipids from animal tissues. J Biol Chem 226: 497-509, 1957.

8. Hsu SM, Raine L and Fanger H: Use of avidin-biotin-peroxidase complex (ABC) in immunoperoxidase techniques: a comparison between $\mathrm{ABC}$ and unlabeled antibody (PAP) procedures. J Histochem Cytochem 29: 577-580, 1981

9. den Boer M, Voshol PJ, Kuipers F, Havekes LM and Romijn JA: Hepatic steatosis: a mediator of the metabolic syndrome. Lessons from animal models. Arterioscler Thromb Vasc Biol 24: 644-649, 2004.

10. Bacon BR, Farahvash MJ and Janney CG: Nonalcoholic steatohepatitis: an expanded clinical entity. Gastroenterology 107: 1103-1109, 1994.

11. Scoppola A, Montecchi FR, Menzenger G and Lala A: Urinary mevalonate excretion rate in type 2 diabetes: role of metabolic control. Atherosclerosis 1565: 357-361, 2001.

12. Simonen P, Gylling H, Howard AN and Miettinen TA: Introducing a new component of the metabolic syndrome: low cholesterol absorption. Am J Clin Nutr 72: 82-88, 2000.

13. Malaisse WJ, Like AA, Malaisse-Lagae F, Gleason RE and Soeldner JS: Insulin secretion in vitro by the pancreas of the sand rats (Psammomys obesus). Diabetes 17: 752-759, 1968.

14. Kaiser N, Yuli M, Üçkaya G, Oprescu AI, Berthoult M-F, Kargar C, Donath MY, Cerasi E and Ktorza A: Dynamic changes in $\beta$-cell mass and pancreatic insulin during the evolution of nutrition-dependent diabetes in Psammomys obesus. Diabetes 54: 138-145, 2005.

15. Malaisse WJ, Malaisse-Lagae F and Wright PH: Effect of fasting upon insulin secretion in the rat. Am J Physiol 213: 843-848, 1967. 